\title{
DESCRIPCIÓN DE UNA NUEVA ESPECIE DEL GÉNERO PSEUDOTRIARTHRON NORMAND, 1938 PARA LA PENÍNSULA IBÉRICA (COLEOPTERA, LEIODIDAE, LEIODINAE)
}

\author{
J. A. Sáez Bolañoํ, J. M. Blanco Villero² \& J. L. Lencina ${ }^{3}$
}

\section{RESUMEN}

J. A. Sáez Bolaño, J. M. Blanco Villero \& J. L. Lencina. 2013. Descripción de una nueva especie del género Pseudotriarthron Normand, 1938 para la península ibérica (Coleoptera, Leiodidae, Leiodinae). Grael/sia, 69(1): 7-16.

Se describe una nueva especie de Pseudotriarthron Normand, 1938 de la península ibérica. Los especímenes de la serie tipo proceden de Diapiro de la Rosa (Jumilla, Murcia). El género Pseudotriarthron fue creado por Normand en 1938 para $P$. numidicum para separarlo del género Triarthron Märkel, 1840. Previamente Wollaston había creado en 1857 el género Stereus para $S$. cercyonides. Ulteriormente se describió Triarthron thurepalmi (Israelson, 1978). Daffner (1983) unificó las tres especies, pero lo hizo bajo el género Stereus, género preocupado por Stereus Mannerheim, 1846 y por tanto no utilizable. Agrupamos ahora las tres especies más la que se describe bajo el género Pseudotriarthron Normand, 1938, que no ha vuelto a ser utilizado. Pseudotriarthron hispanicum n. sp. participa del conjunto de caracteres que tipifican dicho género. En la actualidad se conocen únicamente tres especies de Pseudotriarthron: P. cercyonides (Wollaston, 1857), P. numidicum Normand, 1938 y $P$. thurepalmi (Israelson, 1978). De ellas, únicamente $P$. thurepalmi está bien caracterizada, pues los Tipos de las otras dos están perdidos y no se conocen otros ejemplares, por lo que únicamente contamos con las descripciones originales. No obstante es posible separar con claridad Pseudotriarthron hispanicum n. sp. no sólo de S. thurepalmi, sino también de $S$. cercyonides y de $S$. numidicum tomando como base los caracteres morfológicos que se detallan en la Discusión.

Palabras clave: Coleoptera; Leiodidae; Leiodinae; Pseudotriarthron; especie nueva; península ibérica, España.

\section{ABSTRACT}

J. A. Sáez Bolaño, J. M. Blanco Villero \& J. L. Lencina. 2013. New species of the genus Pseudotriarthron Normand, 1938 from the Iberian Peninsula (Coleoptera, Leiodidae, Leiodinae). Grael/sia, 69(1): 7-16.

We describe a new species of Pseudotriarthron Normand, 1938 from the iberian peninsula. Specimens of the type series are from Diapiro de la Rosa (Jumilla, Murcia). The genus Pseudotriarthron was created by Normand in 1938 for P. numidicum, to separate it from

Apdo. 25; 06280 Fuentes de León (Badajoz).

Apdo. 42; 11100 San Fernando (Cádiz); blancovillero@gmail.com

Departamento de Zoología, Facultad de Veterinaria, Universidad de Murcia. Apdo. 4021.30071 Murcia. j1l@um.es 


\begin{abstract}
genus Triarthron Markel, 1840. Wollaston had previously created in 1857 the genus Stereus for S. cercyonides. Triarthron thurepalmi (Israelson, 1978) subsequently was described. Daffner (1983) unified the three species, but did so under the genus Stereus, previously occupied by Stereus Mannerheim, 1846 and therefore unusable. Three species now grouped besides that described in the genus Pseudotriarthron Normand, 1938, which has not be used again. Pseudotriarthron hispanicum $\mathbf{n}$. sp. shares the set of characters that typify the genre. At present only three species are known: $P$. cercyonides (Wollaston, 1857), $P$. numidicum Normand, 1938 y $P$. thurepalmi (Israelson, 1978). Of these, only $P$. thurepalmi is well characterized, since the Types of the other two are lost and no other specimens are known, so that we have only the original descriptions. However it is possible to clearly separate Pseudotriarthron hispanicum n. sp. not only from of Pseudotriarthron thurepalmi but also from $P$. cercyonides and $P$. numidicum based on the morphological features detailed in the Discussion.
\end{abstract}

Key words: Coleoptera; Leiodidae; Leiodinae; Pseudotriarthron; new species; iberian peninsula; Spain.

\section{Introducción}

En 1938 Normand crea el género Pseudotriarthron para P. numidicum de Túnez, para separarlo del género Triarthron Märkel, 1840. Previamente Wollaston había creado en 1857 el género Stereus para $S$. cercyonides de Madeira. Entre los caracteres que Wollaston utilizó para separarlo del género más afín, es decir Triarthron, podemos citar fundamentalmente las tibias dilatadas y fuertemente espinulosas y los ojos que, en visión dorsal, no sobresalen apenas del contorno de la cabeza. Más recientemente Israelson, en 1978, describe Triarthron thurepalmi de las Islas Canarias. Daffner (1983) con buen criterio y tras estudiar los caracteres morfológicos de estas tres especies, las reagrupa bajo el género Stereus, sin percatarse de que este género estaba preocupado por Stereus Mannerheim, 1846 -un género de Curculionidae-y por tanto no utilizable (a su vez Stereus Mannerheim, 1846 es sinónimo junior de Sthereus Motschulsky, 1845). Agrupamos ahora las tres especies más la que se describe bajo el género Pseudotriarthron, género que no ha vuelto a ser utilizado. Pseudotriarthron hispanicum n. sp. participa del conjunto de caracteres que tipifican dicho género, caracteres que fueron unificados por Daffner (1983).

En consonancia con lo mencionado, se proponen nuevas combinaciones, de tal forma que las cuatro especies conocidas del género quedarían como sigue:

Pseudotriarthron cercyonides (Wollaston, 1857) comb. nov.

= Stereus cercyonides Wollaston, 1857

Pseudotriarthron numidicum Normand, 1938
Pseudotriarthron thurepalmi (Israelson, 1978) comb. nov.

= Triarthron thurepalmi Israelson, 1978.

Pseudotriarthron hispanicum $\mathbf{n}$. sp.

\section{Material y Métodos}

Se han estudiado un total de doce ejemplares de la especie que se describe, todos procedentes de Diapiro de la Rosa (Jumilla, Murcia, España). Los ejemplares de la nueva especie capturados en 1990, fueron colectados atraídos con trampa de luz mixta, mientras que los capturados en 2011 se obtuvieron con trampas de caída sin cebar, abiertas al raso y conteniendo como líquido conservante agua saturada con sal común.

El estudio morfológico se efectuó sobre los ejemplares en seco mediante la observación con microscopio estereoscópico. Para la extracción de las genitalias de los machos fue necesaria la rehidratación de los ejemplares, siendo éstas montadas entre portaobjetos y cubreobjetos con resina DMHF, salvo una que quedó en la misma cartulina. Las mediciones fueron efectuadas mediante un ocular micrométrico.

Las microfotografías han sido tomadas con una cámara digital acoplada al estereomicroscopio. Los dibujos son originales de uno de los autores (J.A.S.B).

\section{Resultados}

\section{Pseudotriarthron hispanicum n. sp.}

SERIE TIPO: Holotypus: Porta cuatros etiquetas identificativas; una impresa con el símbolo de sexo macho; una blanca manus- 
crita: anverso «Jumilla / Murcia / J. L. Lencina leg»; reverso: «Diapiro de la Rosa / 04- VI- 90»; la segunda etiqueta roja e impresa: «Holotypus / Pseudotriarthron hispanicum n. sp. / Sáez, Blanco \& Lencina det. 2011».

PARATYPI: Once ejemplares; identificados todos con las mismas tres etiquetas: una blanca impresa indicativa de sexo: otra blanca impresa: «Diapiro de la Rosa / Jumilla / Murcia / J. L. Lencina leg / fecha»; la segunda etiqueta roja e impresa: «Paratypus / Pseudotriarthron hispanicum n. sp. / Sáez, Blanco \& Lencina det. 2011», variando únicamente las fechas que son las siguientes: 2 machos y 6 hembras de 4-VI-90; 1 macho y dos hembras del 8-V al 18-VI-11.

Tanto el Holotipo como cuatro paratipos quedan depositados en el Museo Nacional de Ciencias Naturales de Madrid; dos parejas en la colección JLL (Jumilla, Murcia) y otra en la colección JMBV (San Fernando, Cádiz).

LOCALIDAD TíPICA: España, Murcia, Jumilla, Diapiro de la Rosa, 680 m, UTM: 30SXH5456.

DesCripción DEL holotipo (Fig. 1): Forma general oval y muy convexa. La longitud total, tomada desde el extremo de las mandíbulas al ápex elitral, es de $2,7 \mathrm{~mm}$. Anchura máxima es de 1,2 mm. Coloración uniformemente amarillenta dorada, algo más oscura por debajo con la maza antenar y la zona anterior de las mandíbulas aún más oscuras.

Cabeza (Fig. 2): Grande, transversa, casi el doble de ancha que de larga, con una longitud de $0,35 \mathrm{~mm}$ y una anchura de $0,75 \mathrm{~mm}$. Posee un reborde anterior que arranca del borde anterior de los ojos para desaparecer en el centro del margen anterior del clípeo que, además, aparece escotado a ese nivel. Existe un fuerte declive clípeofrontal delimitado por una franja negra que no es más que la transparencia de la base de las mandíbulas. Labro fuertemente escotado. Mandíbulas grandes y fuertes, de una longitud de $0,31 \mathrm{~mm}$ (L/A en la base: 1); la izquierda con un único diente apical y otro, obtuso, en medio del borde interno; la derecha con un diente apical bifurcado y un diente agudo en el tercio basal del borde interno que acaba en dos cúspides; entre ambos existe una concavidad superior donde encaja el diente de la mandíbula izquierda (Fig. 2). Ojos poco salientes y relativamente pequeños en visión dorsal. Punteado fino y esparcido, poco profundo, con una distancia entre los puntos 5 o 6 veces su diámetro, con el tegumento entre los puntos brillante y sin microescultura. En los márgenes se aprecia algunos pelos finos y relativamente largos.

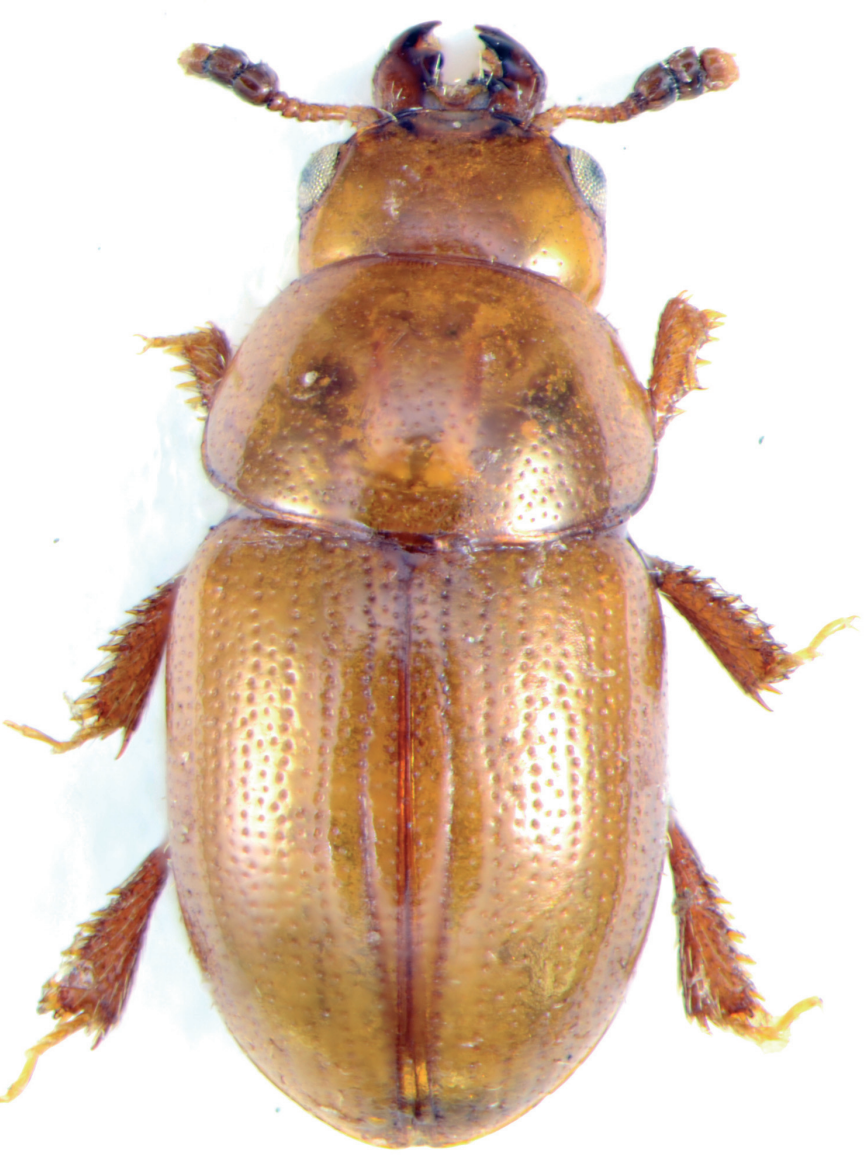

Fig. 1.- Pseudotriarthron hispanicum n. sp.: Habitus en visión dorsal del holotipo.

Fig. 1.- Pseudotriarthron hispanicum n. sp.: Dorsal view of the holotype.

Antenas de once artejos, los tres últimos formando una maza con una longitud total de 0,55 $\mathrm{mm}$, de los cuales $0,25 \mathrm{~mm}$ corresponden a la maza (Fig. 2). Las dimensiones de los artejos son las siguientes (LxA, en micras): $1^{\circ}: 75 \times 50 ; 2^{\circ}: 50 \mathrm{x}$ $45 ; 3^{\mathrm{o}}: 50 \times 27,5 ; 4^{\mathrm{o}}: 25 \times 32,5 ; 5^{\mathrm{o}}: 25 \times 32,5 ; 6^{\mathrm{o}}: 25$ x 45; $7^{\circ}: 25 \times 50 ; 8^{\circ}: 25 \times 50 ; 9^{\circ}: 80 \times 100 ; 10^{\circ}: 70$ $\mathrm{x} 125 ; 11^{\circ}: 100 \times 100$. Palpos maxilares con el último artejo recto y algo más corto que los dos primeros reunidos.

Pronoto (Fig. 1): Es transverso, con una longitud de $0,7 \mathrm{~mm}$ y un anchura máxima, situada por detrás del medio y cerca de los ángulos posteriores, de $1,05 \mathrm{~mm}$ y claramente rebordeado en todo su contorno. Ángulos anteriores redondeados. Los ángulos posteriores son casi indistintos, de 
2
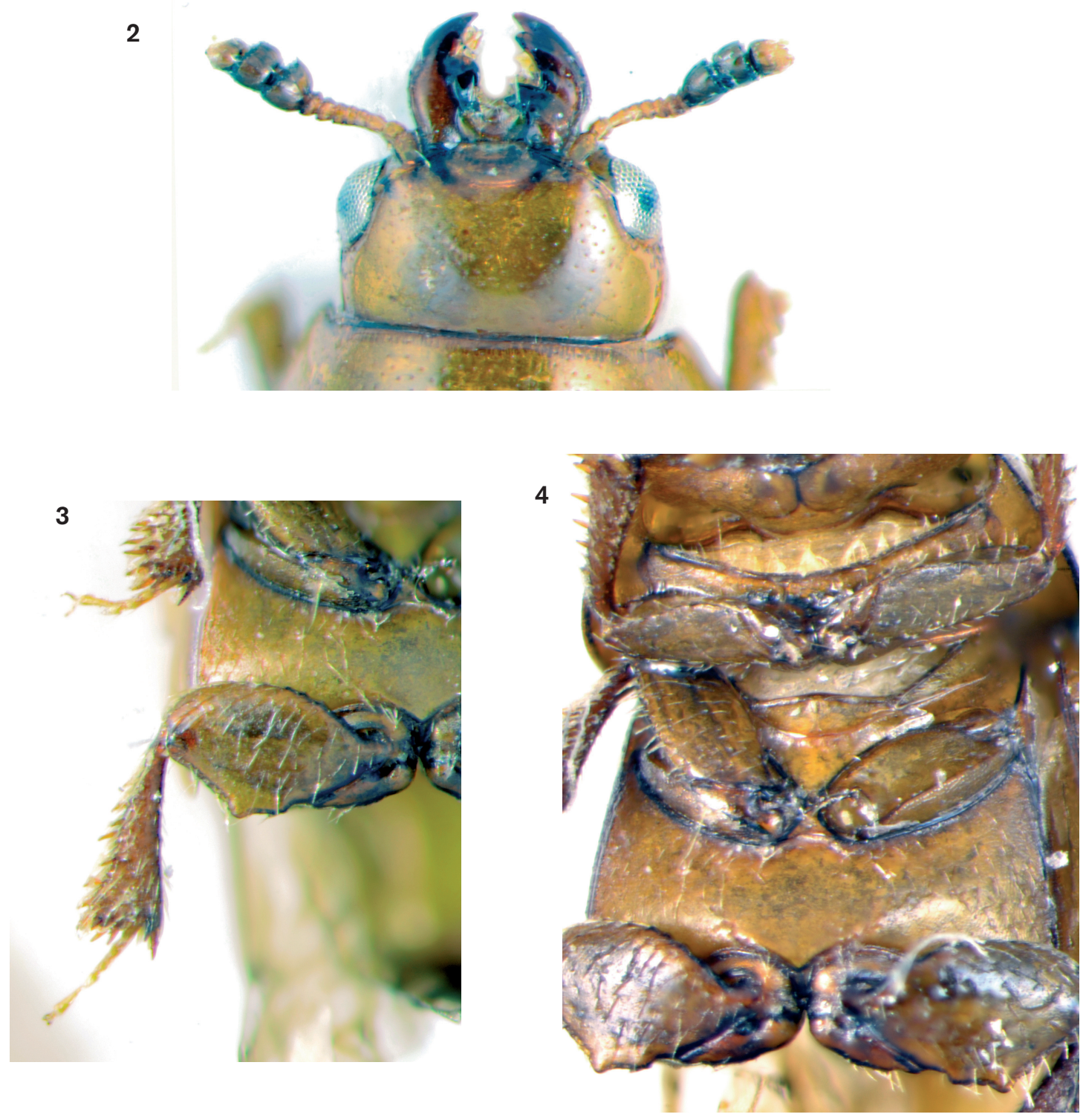

Figs. 2-4.- Pseudotriarthron hispanicum n. sp.: 2) Vista dorsal de la cabeza, antenas y mandíbulas. 3) Metafémur. 4) Visión ventral del macho (paratipo).

Figs. 2-4.- Pseudotriarthron hispanicum n. sp.: 2) Dorsal view of the head, antennae and mandibles. 3) Metafemur. 4) Ventral view of the male (paratype).

tal manera que los bordes laterales se continúan de modo insensible con el borde posterior en curva regular. Punteado fino y poco denso, integrado por puntos algo más fuertes que los de la cabeza, pero de la mitad del diámetro que los de los élitros; su diámetro es mucho menor que la distancia que los separa. Tegumento entre los puntos brillantes y sin microescultura. En los márgenes se aprecia una pilosidad fina, erguida y espaciada.

Escudete: Pequeño y triangular de base ancha, escasamente visible dorsalmente.

Élitros (Fig. 1): Longitud de 1,4 mm y un ancho conjunto de $1,2 \mathrm{~mm}$. que se obtiene cerca del ángulo humeral. Las estrías, en número de nueve, aparecen formadas por hileras de puntos fuertes y 


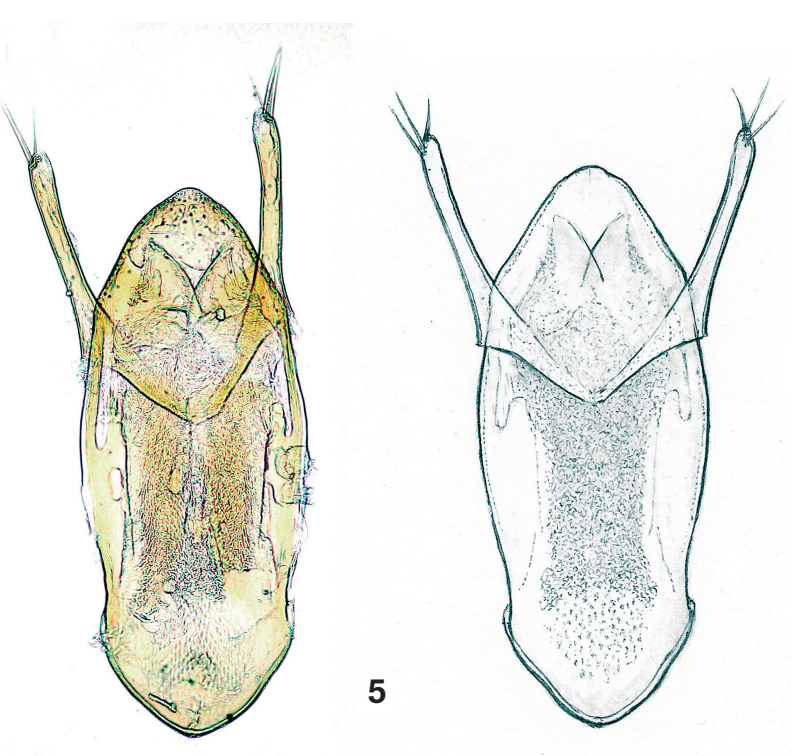

6

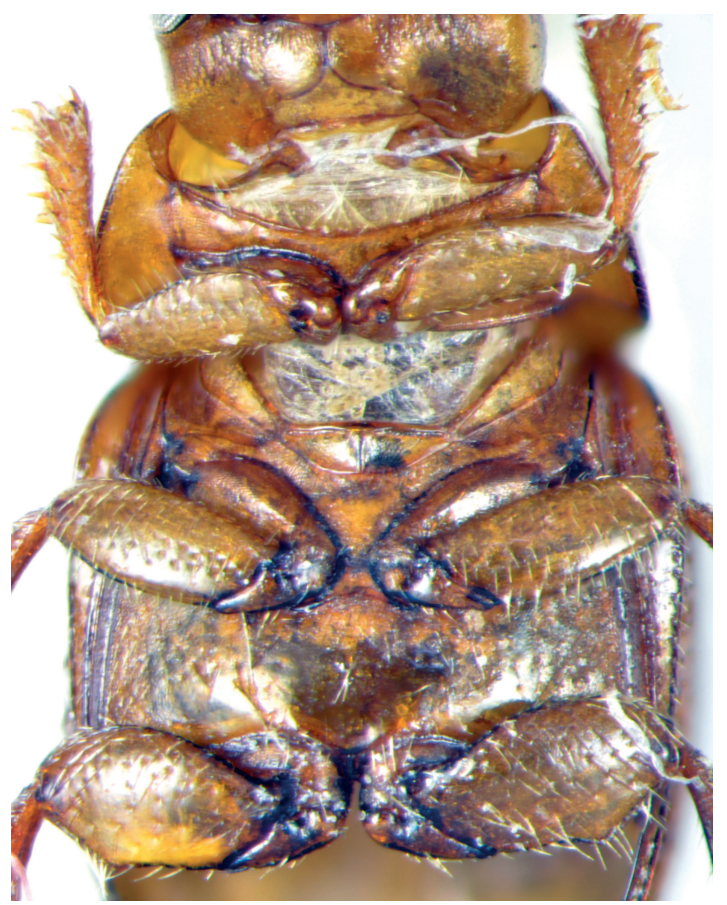

Figs. 5-6.- Pseudotriarthron hispanicum n. sp.: 5) Fotografía (izquierda) y dibujo (derecha) del edeago en visión dorsal. 6) Visión ventral de la hembra (paratipo).

Figs. 5-6.- Pseudotriarthron hispanicum n. sp.: 5)Photography (left) and drawing (right) of the aedeagus in dorsal view. 6) Ventral view of the female (paratype).

gruesos, cuyo diámetro es prácticamente igual que la distancia que los separa. La primera estría en el disco se ensancha para incurvarse progresivamente hacia la sutura elitral, desvaneciéndose en el declive. Desde el disco hasta el declive aparece hundida, configurando una auténtica estría. En el centro de lo que podríamos llamar interestrías, que son planas, se aprecia otra hilera de puntos alineados pero más finos que los de las estrías, aproximadamente de la mitad de su diámetro. El tegumento entre los puntos es brillante y sin microescultura. Entre la octava y novena interestría se aprecia una pilosidad escasa, a base de pelos finos, alineados y relativamente largos. En el resto de la superficie elitral, principalmente en el declive, se observan algunos cilios aislados. Epipleuras bien desarrolladas que van estrechándose hacia atrás, hasta desaparecer cerca del ápex. Especie macróptera.

Patas: Los metafémures son muy anchos y están provistos de un diente muy marcado en el centro de su borde posterior y otro, menos marcado y más medial, que delimitan dos sinuosidades entre el diente y el trocánter (Fig. 3). Además muestra largos pelos. Las tibias se ensanchan progresivamente hacia el ápex, adoptando una forma de espátula. Borde externo provisto de seis u ocho gruesas y fuertes espinas, además de las apicales. En la cara inferior de las tibias medias y posteriores existen espinas similares; entre estas espinas se observa una fuerte pilosidad. Tarsos finos y comprimidos lateralmente, con una fórmula de 5-5-5.

Región ventral del cuerpo algo menos brillante que el dorso. Suturas gulares reunidas y separándose hacia atrás en forma redondeada. Gula triangular. Mesoventrito no punteado, dividido en dos porciones por una sutura careniforme transversal y semicircular de concavidad anterior que va desde una sutura pleurosternal mesotorácica a la otra. En el borde anterior se aprecia un engrosamiento en forma de tubérculo escotado. Entre el tubérculo y la carena se aprecia un discreto engrosamiento medial longitudinal (Fig. 4). Metaventrito prácticamente 
plano y no punteado, lampiño en el centro y con cilios escasos y largos en las porciones laterales. Muestra una sutura careniforme anterior que delimita el proceso metaventral. A ese nivel, el metaventrito muestra una leve depresión. Uroventritos iguales entre sí y provistos de una pilosidad tumbada hacia atrás.

Edeago (Fig. 5). De morfología relativamente simple, aparece integrado por un lóbulo medio relativamente ancho (relación L/A: 2,4), muy redondeado en la base y discretamente apuntado en el ápice; muy poco curvado ventralmente. Por transparencia el saco interno muestra dos dientes apicales triangulares solapados en su porción inferior, apreciándose un denso fascículo de espínulas casi hasta la base. Los estilos laterales sobrepasan el ápice del lóbulo medio y aparecen unidos entre sí formando un ángulo obtuso formando una pieza única; el ápice de los mismos muestra tres cerdas.

DIMORFISMO SEXUAL: Las hembras poseen unos metafémures mucho más estrechos con el borde posterior formando un ángulo obtuso en el centro que no configura un auténtico diente y que no delimita sinuosidad alguna (Fig. 6).

VARIABILIDAD: En lo que al tamaño respecta no existen diferencias apreciables; los tres machos tienen una media de longitudes totales de $2,80 \mathrm{~mm}$ $(2,70 ; 2.95$ y 2,75$)$; mientras que los de las siete hembras nos da una media de $2,65 \mathrm{~mm}$, sin embargo una de las hembras es el mayor ejemplar de la serie con $3,05 \mathrm{~mm}(2,30 ; 2,70 ; 2,30 ; 2,65 ; 2,80$; $2,75$ y 3,05$)$. La relación L/A se mantiene entre 2,09 y 2,25 . Todos los especimenes muestran la misma coloración; sin embargo algunas hembras tienen el último artejo antenar más claro que los del resto de la maza.

ETIMOLOGÍA: El nombre elegido es el adjetivo latino hispanicum, relativo o perteneciente a Hispania, dado que se trata de la primera especie de este género en España y, por ende, de la península ibérica y del continente europeo.

\section{Discusión}

Las características del género Pseudotriarthron fueron definidas por Normand (1938) y sistematizadas por Daffner (1983) quien unificó las tres especies conocidas hasta ese momento, teniendo en cuenta la descripción de Stereus (Wollaston, 1857), género en el que las agrupó. Pseudotriarthron se caracterizaría por la siguiente combinación de caracteres: 1) Tarsos posteriores delgados, filiformes, con una fórmula 5-5-5. 2) Mandíbulas cerradas con forma ojival característica; la izquierda con dos dientes separados por una profunda hendidura; la derecha con tres dientes. 3) Maxila y labro con palpo. 4) Clípeo separado de la frente por un surco neto en forma de arco. 5) Ojos que, en visión dorsal, apenas sobresalen del contorno de la cabeza, no presentando estrechamiento alguno por detrás del mismo. 6) Cabeza desprovista de surco antenar. 7) Mesosterno plano no aquillado e inclinado hacia atrás. 8) Tibias ensanchadas en el extremo y fuertemente espinuladas en el borde externo; las anteriores largas y con un profundo surco para alojar los tarsos. 9) Epipleuras con una pilosidad blanquecinoamarillenta.

El género Pseudotriarthron se diferenciaría del género Deltocnemis J. Sahlberg, 1886 en que éste último posee una fórmula tarsal de 4-4-4 y tienen la base del pronoto fina pero claramente rebordeada, carácter que comparte con los géneros Sogda Lopatin, 1961, Hydnobius W.L.E. Schmidt, 1841 y Triarthron Märkel, 1840. Con este último género comparte el carácter de poseer una maza antenar compuesta por tres artejos, pero se diferencia por tener los artejos de los tarsos posteriores ensanchados distalmente y los ojos que sobresalen claramente del contorno de la cabeza.

Ciñéndonos al género Pseudotriarthron reconocemos actualmente tres especies además la que aquí se describe: P. cercyonides (Wollaston, 1857) de Madeira, P numidicum Normand, 1938 de Túnez y P. thurepalmi (Israelson, 1978) de Tenerife (Islas Canarias) (Daffner, 1983, Perreau, 2004).

\section{Pseudotriarthron cercyonides (Wollaston, 1857) comb. nov.}

Si bien Wollaston (1857) ya indica que la especie es muy rara, Daffner (1983) no pudo estudiar ningún ejemplar pues no logró encontrarlos en el Museo Británico, que es donde él suponía que debían estar. Sin embargo la colección de Wollaston fue adquirida -entre otras muchas- por René Oberthür; a la muerte de este último la colección fue vendida por la familia al Museo de Historia Natural de París (Cambefort, 2006). Al igual que le sucedió a Daffner, a pesar de todos nuestros esfuerzos no 


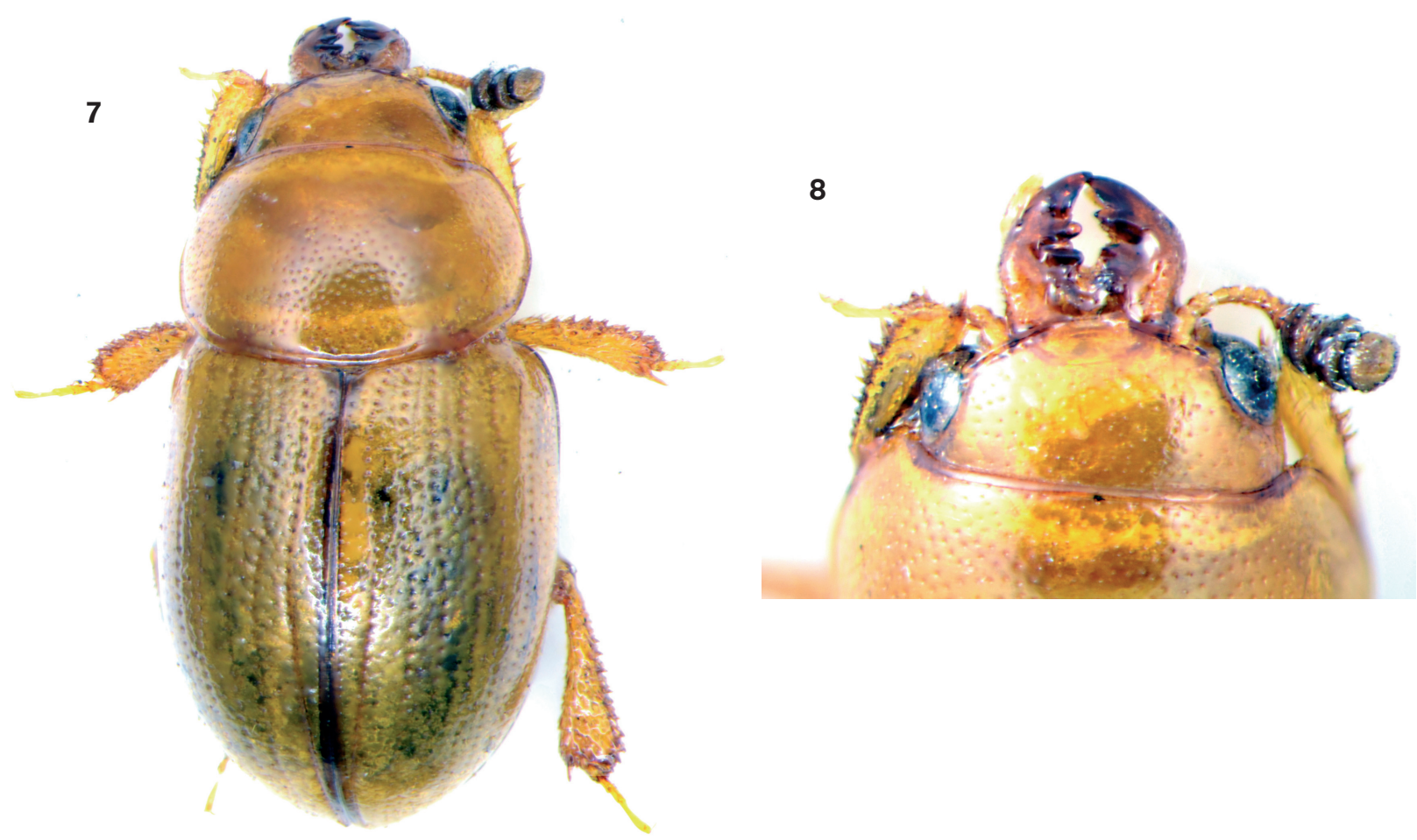

Figs. 7-8.- Pseudotriarthron thurepalmi (Israelson, 1978): 7) Habitus en visión dorsal. 8) Vista dorsal de la cabeza, antenas y mandibulas.

Figs. 7-8.- Pseudotriarthron thurepalmi (Israelson, 1978): 7) habitus, dorsal view. 8) Dorsal view of the head, antennae and mandibles.

hemos podido obtener ni un solo ejemplar de comparación. Sin embargo la descripción original es bastante aceptable y de este modo, Pseudotriarthron hispanicum n. sp. se separa fácilmente de $P$. cercyonides porque esta especie tiene el tegumento alutáceo y es de color negro o negro-píceo, mientras $P$. hispanicum n. sp. lo tiene brillante y es amarillo.

Pseudotriarthron thurepalmi (Israelson, 1978) comb. nov.

Fue descrito en 1978 por Israelson sobre un único ejemplar macho de Santa Cruz de Tenerife (Thure Palm leg. IV/1967). Daffner vió únicamente el Tipo en la colección Palm. Más recientemente se han capturado tres ejemplares, dos machos y una hembra en la Palma (Fuente de Guaidin, 19/II/1984, García, 1986; Machado \& Oromí, 2000) y gracias a la amabilidad de Rafael García y Pedro Oromí hemos tenido acceso a ambos machos. Esta especie está bien caracterizada y también puede separarse con facilidad de P. hispanicum n. sp. por los caracteres que a continuación se detallan. Pseudothriarthron thurepalmi es proporcionalmente más rechoncho (L/A: 2,04$)$ y convexo, tanto en visión dorsal como lateral, con la anchura máxima en la porción media de los élitros (Fig. 7). La cabeza muestra el borde anterior del clípeo apenas sinuado, casi semicircular y las mandíbulas son de estructura diferente, más robustas: la izquierda con un diente apical y otros dos dientes, aqlgo romos, perpendiculares al eje en el centro del borde interno; la derecha con un diente apical bifurcado y otro más basal y romo (Fig. 8). El pronoto exhibe su máxima anchura en el medio, con un punteado más fuerte y denso y el reborde posterior es más grueso. Los élitros muestran un punteado de las estrías más fuerte; por el contrario los puntos de las interestrías son más finos, menos alineados y más escasos; la primera estría está más 

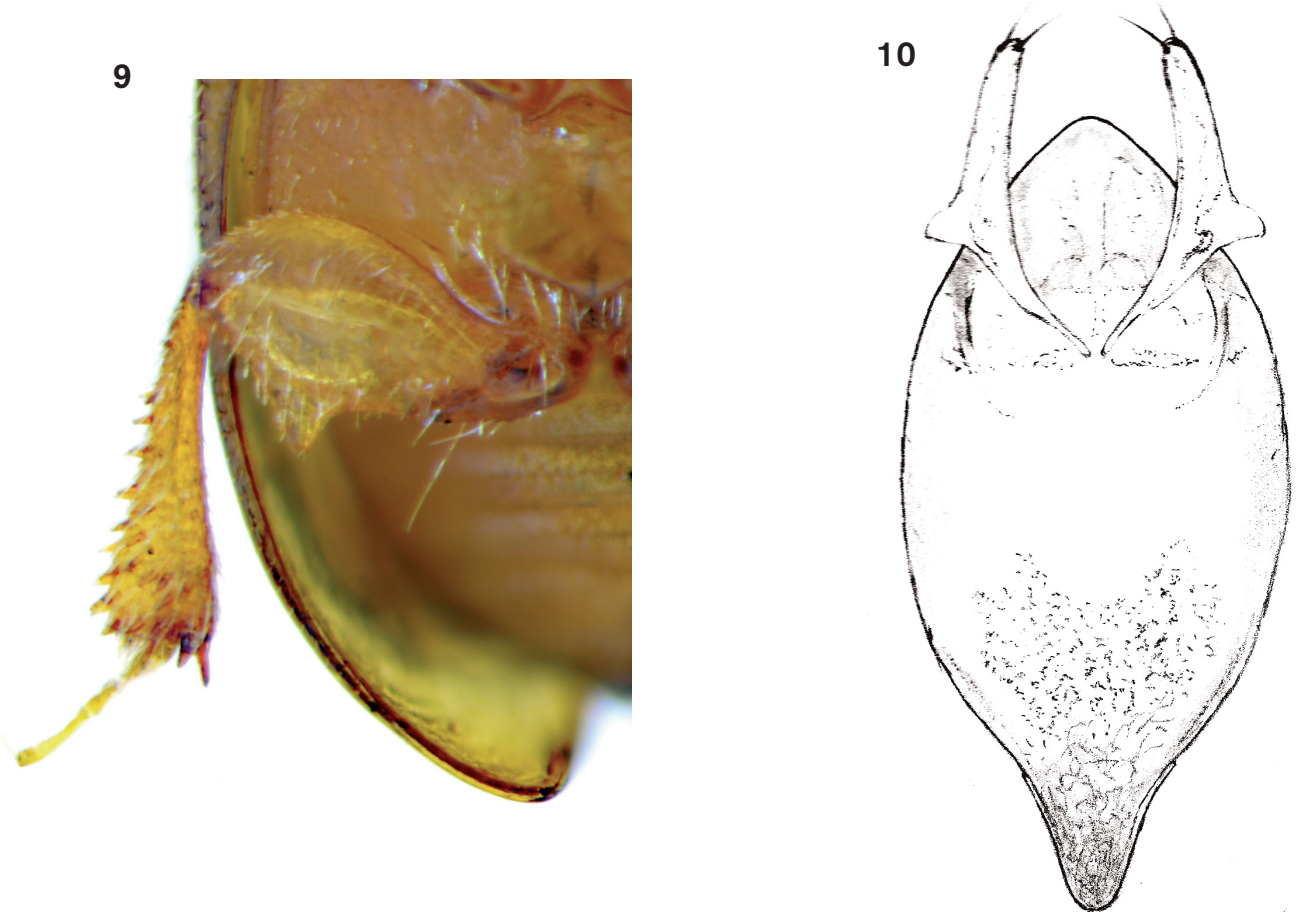

Figs. 9-10.- Pseudotriarthron thurepalmi (Israelson, 1978): 9) Metafémur del macho. 10) Edeago.

Figs. 9-10.- Pseudotriarthron thurepalmi (Israelson, 1978): 9) Metafémur of the male. 10) Aedeagus.

sinuada hacia afuera en el disco, originando una anchura mayor de la primera interestría a ese nivel. Los fémures posteriores del macho muestran un único diente triangular muy prominente (Fig. 9). El edeago muestra una estructura general similar; sin embargo el lóbulo medio aparece muy acuminado en su base y proporcionalmente más ancho en el medio. Los estilos laterales son proporcionalmente más cortos y de mayor grosor y forman un codo saliente en la vertiente externa a nivel de la unión entre el estilo propiamente dicho y el arranque del arco (Fig. 10).

\section{Pseudotriarthron numidicum Normand, 1938}

Esta especie fue descrita por Normand (1938) sobre un único ejemplar "un poco inmaduro" capturado en septiembre de 1937 en Le Kef (Túnez). Daffner (1983) no pudo ver el Holotipo, a pesar de los muchos esfuerzos que realizó y tampoco pudo ver ningún otro ejemplar. Dice textualmen- te: "la identidad de esta especie solo podrá aclararse cuando esté disponible para su examen" (traducción del alemán). A pesar de todos nuestros esfuerzos, no ha sido posible obtener ni un solo ejemplar de comparación. En principio sería la especie mas afín a $P$. hispanicum n. sp., pero disponemos de la descripción que afortunadamente es muy detallada por lo que, basándonos en ella, se separaría fundamentalmente porque $P$. numidicum muestra la máxima anchura del pronoto por delante del medio, mientras $P$. hispanicum n. sp. muestra la máxima anchura por detrás del medio, cerca de los ángulos posteriores. Además, en $P$. numidicum el primer artejo antenar es dos veces más largo que ancho y los palpos maxilares tienen el último artejo un poco recurvado y tan largo como los dos anteriores reunidos, mientras que en $P$. hispanicum n. sp. el primer artejo antenar es una vez y media más largo que ancho y en los palpos el último artejo no está recurvado y es algo más corto que los dos precedentes reunidos. 
NotAs AUTOECOLÓGICAs: No se conoce prácticamente nada acerca de las costumbres de esta especie ni de ninguna otra del género.

Wollaston (1865) indica, refiriéndose a $P$. cercyonides, que esta especie es «Peculiar to the sylvan regions in Madeira proper, where it burrows beneath fallen leaves and other vegetable refuse lying on the damp ground - a mode of life for which its robust, spinose tibiae eminently fit it. It is extremely rare, and was detected by myself during the summer of 1885 in the laurel-woods at the head of the Santa Cruz ravine, at San Antonio da Serra. And I subsequently met with it, under decaying rubbish, at the Lombarda das Vacas (on the mountains to the east of Sao vicente». Por su parte Normand (1938) indica refiriéndose a $S$. numidicum que la especie fue hallada en «Le Kef, un exemplaire un peu immature, capturé, en septembre 1937, dans les détritus charries par l'oued Remel».

García (1986) capturó sus ejemplares de $P$. thurepalmi tamizando un tocón putrefacto de laurel en el mes de febrero.

Nuestros ejemplares han sido capturados en El Diapiro de la Rosa. Se trata de una unidad singular situada en el extremo occidental del parque regional de la Sierra del Carche, bien caracterizada por tratarse de un diapiro salino que emerge de la llanura cultivada. Los materiales extrusionados corresponden a arcillas y margas de colores variegados, yeso, sal y dolomías del Triásico. La acción diapírica de estos materiales se sigue produciendo en nuestros días, lo que ha contribuido a que se preserve una perfecta morfología en domo, alargado en sentido NO-SE. Los suelos poseen una elevada salinidad y un perfil poco desarrollado, correspondiendo a Solonchaks litosólicos asociados a Litosoles, con inclusiones de Xerosoles gípsicos (B.O.R.M., 2002).

La vegetación está dominada por comunidades gipsícolas bien estructuradas, básicamente tomillares del Teucrio libanitis-Thymetum membranacei en los suelos más degradados, y romerales y espartizales en los mejor conservados, en general con baja cobertura; son también frecuentes las comunidades subnitrófilas dominadas por quenopodiáceas. Donde se acumulan especialmente las sales, se encuentra un albardinal rico en Limonium caesium (Girard) Kuntze. Sobre buena parte del área se han llevado a cabo repoblaciones de Pinus halepensis Mill. mediante aterrazado, lo que ha conducido a una ace- leración de los procesos erosivos y la destrucción de buena parte de la vegetación autóctona, aunque se observa una buena regeneración del tomillar gipsícola. En cuanto al paisaje, predomina el factor abiótico, por sus relieves abruptos y la escasez de cubierta vegetal, destacando también las repoblaciones forestales en terrazas. En esta unidad se detecta escasa actividad humana, quedando restringida a pequeñas manchas de cultivos de almendros de escasa rentabilidad. En el centro de la unidad existe una zona antiguamente cultivada, que hoy es ocupada por vegetación natural (B.O.R.M., 2002).

\section{Clave dicotómica de las especies del género Pseudotriarthron}

1. Toda la superficie del tegumento alutácea; es decir con aspecto de cuero muy fino arrugado. Madeira

$P$. cercyonides

1'. Superficie del tegumento brillante, no alutácea .......... 2

2. Pronoto con punteado moderamente denso y fuerte, con su anchura máxima en el medio. Tenerife (Islas Canarias) P. thurepalmi

2' Pronoto con un punteado fino y esparcido; anchura máxima por delante o por detrás del medio ................ 3

3. Anchura máxima del pronoto por delante del medio (Túnez) $P$. numidicum

3'. Anchura máxima del pronoto por detrás del medio (España) P. hispanicum

\section{Agradecimientos}

A Rafael García y Pedro Oromí quienes amablemente nos han permitido estudiar los dos machos de P. thurepalmi.

A los numerosos colegas a quienes hemos acudido en un intento -desgraciadamente infructuoso- de encontrar ejemplares de comparación de $P$. cercyonides y de $P$. numidicum.

Debemos dar las gracias también al Museo Nacional de Ciencias Naturales de Madrid; muy especialmente a Miguel Ángel Alonso Zarazaga, por sus atinadas sugerencias en lo que a taxonomía se refiere. También a Mercedes París, quien ha puesto a nuestra disposición los fondos de Leiodinae contenidos en las colecciones entomológicas del Museo y a Manuel Sánchez Ruiz, por su apoyo constante y por ayudarnos a localizar bibliografía de difícil acceso.

\section{Referencias}

Boletín Oficial de la Región de Murcia. 2002. Decreto n. ${ }^{\circ} 69$ de 22 de marzo: Plan de Ordenación de los Recursos Naturales de la Sierra de «El Carche», 77: 4767-4807. 
Cambefort, Y., 2006. Des coléoptères, des collections \& des hommes. Publications Scientifiques du Muséum national d'Histoire naturelle. Paris. 375 pp.

Daffner, H., 1983. Revision der paläarktischen Arten der Tribus Leiodini Leach (Coleoptera, Leiodidae). Folia Entomologica Hungarica, 44(2): 9-163.

García, R., 1986. Nuevos datos sobre la distribución de la fauna coleopterológica de Canarias. Vieraea, 16: 73-79.

Israelson, G., 1978. The Leiodini of the Canary Islands (Coleoptera, Leiodidae). Vieraea, [1977], 7(2): 181190.

Lawrence, J. F., Beutel, R. G., Leschen, R. A. B. \& Slipinski, A., 2010. 2. Glossary of Morphological Terms. In: R. A. B. Leschen, R. G. Beutel \& J. F. Lawrence. Handbook of Zoology. Arthropoda: Insecta. Coleoptera, Beetles. Vol. 2: Morphology and Systematics. Walter de Gruyter. Berlin: 9-20.

Machado, A. \& Oromí, P., 2000. Elenco de los coleópteros de las Islas Canarias. Instituto de Estudios Canarios. La Laguna. 306 pp.

Normand, H. 1938. Contribution au catalogue des coléoptères de la Tunisie $\left(13^{\circ}\right.$ fascicule). Premier supplément. Bulletin de la Société d'Histoire Naturelle de l'Afrique du Nord, 29: 337-371.

Perreau, M., 2004. Family Leiodidae. In: I. Löbl \& A. Smetana (eds.). Catalogue of Paleartic Coleoptera. Vol. 2. Apollo Books. Stenstrup: 133-203.
Sáez, J. A., Blanco, J. M. \& Fresneda, J., 2012. Descripción de una nueva especie del género Leiodes Latreille, 1797 de la península ibérica (Coleoptera, Leiodidae, Leiodinae). Graellsia, 68(2): 281-190. doi: 10.3989/graellsia.2012.v68.060

Wollaston, T. V., 1857. Catalogue of the coleopterous insects of Madeira in the collection of the British Museum. British Museum of Natural History. London. 234 pp.

Wollaston, T. V., 1865. Coleoptera Atlantidum being an enumeration of the coleopterous insects of the Madeiras, Salvages, and Canaries. John van Voorst. London. $526+140$ pp. 